\title{
Critical behavior of the single-particle spectral weight in the ionic Hubbard model
}

\author{
M. C. Refolio,* J. M. Lòpez Sancho, and J. Rubio \\ Instituto de Matemàticas y Fìsica Fundamental, CSIC, Serrano 113 bis, E28006 Madrid, Spain \\ (Received 3 December 2004; revised manuscript received 11 March 2005; published 15 July 2005)
}

\begin{abstract}
We show in this paper that the single-particle spectral-weight function (SWF) of the one-dimensional ionic Hubbard model at half filling exhibits critical behavior, as a function of the on-site repulsion $U$, near the chemical potential. The calculation has been carried out within the framework of the cluster perturbation theory approach of Senechal et al., Phys. Rev. Lett 84, 522 (2000). As $U$ increases, the SWF at $k_{F}= \pm \pi / 2$ (the Fermi points) jumps from the two-peak structure (plus two weak side peaks) characteristic of low $U<U_{c}$ to a four-peak structure beyond $U_{c}$ where all the peaks have about the same spectral weight. This abrupt spectralweight transfer from the inner to the outer peaks can be traced to the ionic-neutral transition characteristic of this system and seems to correspond to the first critical point of the two-point scenario discussed in the literature. It is accompanied by a nonvanishing minimum of the single-particle charge gap. As $U$ increases further, the system evolves gradually into a Mott-Hubbard insulator. No other signatures of abrupt change are detected in the SWF. These results are discussed in the light of photoelectron spectroscopic measurements of quasi-one-dimensional materials.
\end{abstract}

DOI: 10.1103/PhysRevB.72.035121

PACS number(s): 71.10.Fd, 71.10.Hf, 71.30.+h

\section{INTRODUCTION}

The ionic Hubbard model (IHM) is just a Hubbard model applied to a bipartite lattice of cations and ions. In one dimension it reads

$$
H=t \sum_{\langle i j\rangle s} c_{i s}^{\dagger} c_{j s}+\frac{\Delta}{2} \sum_{i s}(-1)^{i} n_{i s}+U \sum_{i} n_{i \uparrow} n_{i \downarrow}
$$

with the usual notation, i.e., $c^{\dagger}$ creates while $c$ destroys an electron and $n=c^{\dagger} c$ is an occupation number. Electrons of spin $s(\uparrow$ or $\downarrow$ ) hop among nearest neighbors with a hopping amplitude $t$ and experience a Coulomb repulsion $U$ when two electrons of opposite spin try to occupy the same site. $\Delta$ is the on-site energy difference between even and odd sites, usually known as the charge-transfer energy.

This Hamiltonian provides a simple, minimal model where the interplay among covalency $(t)$, ionicity $(\Delta)$, and correlation $(U)$ gives rise to a rich phase diagram within which different one-dimensional (1D) compounds can be placed. Originally proposed by Nagaosa and Takimoto ${ }^{1}$ as a model for ferroelectric perovskites and later by Egami and co-workers ${ }^{2,3}$ to explain the neutral-ionic transition in some organic crystals, this Hamiltonian is ideal for studying, e.g., the nature of quantum phase transitions in $1 \mathrm{D}$ electron systems. On general grounds, one expects a transition from an ionic, weakly-correlated band insulator (BI) phase to a neutral, strongly-correlated Mott insulator (MI) phase as $U$ increases. An important and controversial issue is the nature of this transition as well as whether two critical points rather than one separate both phases. Depending on the method of calculation used, either one $\mathrm{e}^{4-6}$ or two ${ }^{7-10}$ critical points have been predicted, so that the controversy cannot be considered as completely closed yet, although some consensus is growing in favor of a two-point scenario.

In this paper we shall stay outside this controversy and will instead concentrate on the single-particle spectralweight function (SWF) $A(k, E)$ at half filling. Despite its fun- damental importance, $A(k, E)$ has received little attention regarding this model, most of the effort having been concentrated on the nature of the BI-MI quantum phase transition. $A(k, E)$ will be calculated using the cluster perturbation theory (CPT) approach of Senechal et al. ${ }^{11}$ This is briefly described in Sec II. Section III gives our results for $A(k, E)$ and the density of states followed by a discussion where they are interpreted and related to recent work on quantum phase transitions. Section IV compares with recent photoelectron spectroscopic measurements of quasi-onedimensional (Q1D) materials. Finally, Sec V closes the paper with some concluding remarks.

\section{CLUSTER PERTURBATION THEORY}

Since this method has been discussed at length in Ref. 11, we simply summarize it very briefly here. In CPT one divides the lattice (here the chain) into a number of equal clusters. The single-particle Green's function (GF) for the cluster is then found by exact diagonalization with open boundary conditions. We have made use of a variant of the Lanczos algorithm specifically designed to calculate dynamic quantities. ${ }^{12}$ The approximation now consists in neglecting the intercluster self-energy, so that the GF inverses of neighboring clusters are connected by hopping terms only. Periodic boundary conditions are then imposed on the whole chain, i.e., between the extreme clusters. To be specific, let $m_{i}$ denote the site $m$ of the cluster $i$. The exact GF $G_{m_{i}, n_{j}}$, of the whole chain is given by the well-known Dyson's equation in matrix form $\left(G_{0}^{-1}-\Sigma\right) G=I$, in terms of the noninteracting GF and the exact $\Sigma$. In CPT this exact $\Sigma$ is approximated by $\Sigma_{m_{i}, n_{j}}=\delta_{i j} \Sigma_{m n}^{C}$ where $\Sigma^{C}$ is the cluster self-energy matrix. This approximation is applicable to any lattice in any dimension. It can be understood as a lowest-order contribution to a systematic perturbation expansion in powers of the intercluster hopping. ${ }^{11,13}$ It turns out, on the other hand, that 
CPT is a limiting case of a more general variational cluster approach. $^{14}$

As stated before, we shall concentrate on the SWF $A(k, E)$, which can be compared with angle-resolved electron spectroscopy (ARPES). It is given as usual by

$$
A(k, E)=-\frac{1}{\pi} \operatorname{Im} G(k, E+i \eta)
$$

where $G(k, E+i \eta)$ is the Fourier transform (FT) of the single-particle retarded GF and $\eta$ a small positive number. This FT must be calculated with some care since $G_{m n}(i-j)$ is periodic in $i j$ but not in $m n$ due to the open boundary conditions used in the clusters. The correct formula is ${ }^{11,13}$

$$
G(k, E+i \eta)=\frac{1}{N} \sum_{m n} e^{-i k(m-n)} G_{m n}(N k, E+i \eta)
$$

where $N$ is the number of sites in a cluster and $G_{m n}(k)$ the FT of $G_{m n}(i-j)$. We shall also make use of the single-particle density of states (DOS), given by

$$
N(E)=\frac{1}{M} \sum_{n_{i}} A_{n_{i}}(E)
$$

where $A_{n_{i}}(E)=-(1 / \pi) \operatorname{Im} G_{m_{i}, n_{i}}(E+i \eta)$ and $M$ is the number of sites of the original chain. As is well known, this function can be compared with total, or integrated, photoelectron spectroscopy.

\section{CRITICAL BEHAVIOR OF THE LOW-ENERGY BAND STRUCTURE}

In all our calculations, we consider a chain of 96 sites of two kinds with levels at $\pm \Delta / 2$, at half filling, and take $t=$ $-1 \mathrm{eV}$ and $\Delta=1 \mathrm{eV}$. Clusters of eight sites have been adopted after checking that increasing the cluster size up to 12 sites does not change much the results for the whole chain in the low-energy region.

\section{A. The spectral-weight function at the Fermi surface}

Figure 1 shows $A(k, E)$ at the Fermi points $k_{F}= \pm \pi / 2$ for increasing $U$. A broadening $\eta=0.05 \mathrm{eV}$ has been given to the otherwise $\delta$ functions. Two regimes are observed in the lowenergy region. For small $U$, a two-peak structure, accompanied by two small side peaks, is seen close to the chemical potential $\mu$ (zero reference energy). This structure persists, while the two inner peaks approach each other, up to $U_{1}$ $=2.0775 \mathrm{eV}$. Abruptly, it changes at $U_{2}=2.0780 \mathrm{eV}$, giving rise to a four-peak structure where all the peaks have almost the same spectral weight. It seems as though the inner peaks of the former structure have transferred spectral weight to the outer peaks. A critical $U=U_{c}$, somewhere between $U_{1}$ and $U_{2}$, separates the two regimes. As $U$ further increases, the inner peaks start to separate from each other while the outer peaks also move apart, but more slowly. At the end, for very large $U$, the SWF tends asymptotically to a Mott-Hubbard strong-coupling phase. No further signatures of abrupt change have been detected for the SWF in this large- $U$ re-

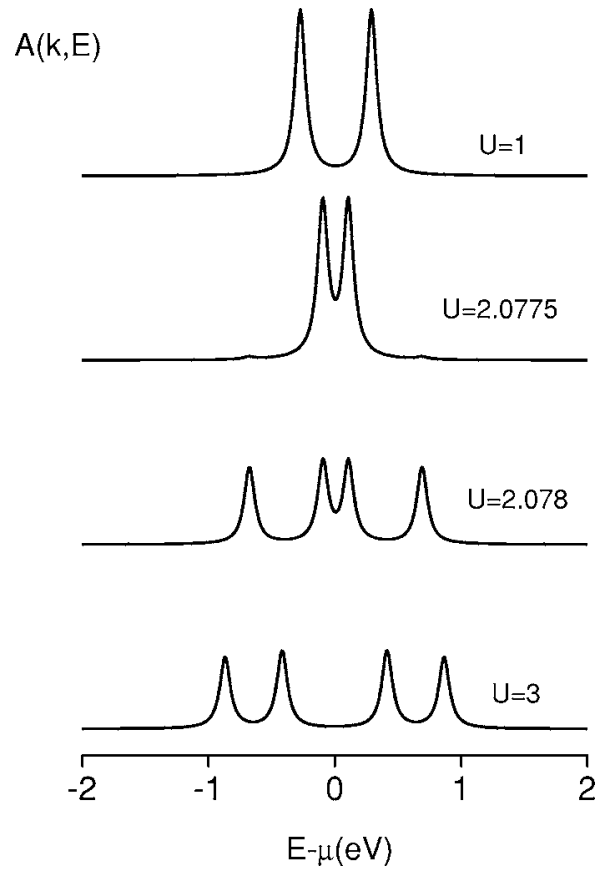

FIG. 1. Single-particle spectral-weight function $A(k, E)$ for the half-filled ionic Hubbard model at the Fermi points $k= \pm \pi / 2$. From top to bottom, $U=1,2.0775$ (just below $U_{c}$ ), 2.078 (just above $U_{c}$ ), and 3 . We have taken $t=-1$ and $\Delta=1$. All the energies are given in $\mathrm{eV}$.

gion. The GF gap, i.e., the single-particle charge gap, after passing through a minimum at $U_{c}$, increases again. It never vanishes, in agreement with recent findings. ${ }^{6,10}$

This change of regime, signaled by an abrupt transfer of spectral weight from the inner to the outer peaks, can be easily traced to the ionic-neutral transition known for many years ${ }^{2,3}$ to take place in the IHM. The noninteracting band structure $\varepsilon_{k}=-2 t \cos k$ vanishes at $k_{F}= \pm \pi / 2$, leading to the isolated atoms limit, with the well-known DOS

$$
N_{i s}(E)=\left(1-\left\langle n_{i-s}\right\rangle\right) \delta\left(E-\Delta_{i}\right)+\left\langle n_{i-s}\right\rangle \delta\left(E-\Delta_{i}-U\right)
$$

where $\left\langle n_{i-s}\right\rangle$ is the average occupation of the $i$ th site with opposite spin and $\Delta_{i}=(-1)^{i} \Delta / 2$. The spin index, implicit everywhere else, is here necessary. It can then be easily seen that

$$
A\left(\frac{\pi}{2}, E\right)=\frac{1}{M} \sum_{i} N_{i}(E)=\frac{N_{1}(E)+N_{2}(E)}{2} .
$$

In general one expects four levels, $\pm \Delta / 2$ and $\pm \Delta / 2+U$. For $U<\Delta$, the odd sites are doubly occupied while the even sites are empty, just the ionic configuration. We therefore have only two levels, $\Delta_{\text {odd }}+U=-\Delta / 2+U$ and $\Delta_{\text {even }}=\Delta / 2$ (the inner levels) both with weight 0.5 , the other two levels, $-\Delta / 2$ and $\Delta / 2+U$ (the outer levels) having zero weight. For $U$ $>\Delta$, however, both even and odd sites are half occupied (either one $\uparrow$ or one $\downarrow$ electron), just the neutral configuration. The four levels, $\pm \Delta / 2$ and $\pm \Delta / 2+U$, have then the same weight $(0.25)$. The abrupt transfer of weight is thus clear. 


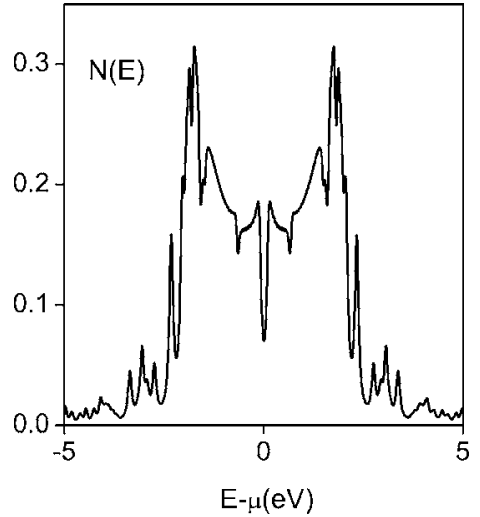

FIG. 2. Density of states for the same model of Fig. 1 with $U$ $=2.0775$, just below $U_{c}$.

One thus would expect a steep drop in both the ionicity $\left(n_{1}-n_{2}\right)$ and the double occupancy as $U$ goes through $\Delta$. When these quantities are calculated from the GF of the whole chain, however, they turn out to be continuous, decreasing functions of $U$, even around $U_{c}$. Moreover, the transition takes place at a value of $U$ more than twice $\Delta$. This means that some compensating changes must have taken place for other $k \neq \pm \pi / 2$. In order to check that $A\left(k_{F}, E\right)$ provides the driving mechanism for the transition, we have performed calculations for chains of $M=4 n+2$ where $n$ is a positive integer. No trace of the abrupt spectral-weight transfer was found. This takes place only in chains of $4 n$ sites, which have special inversion symmetry properties.

\section{B. Density of states and band structure}

Let us first study the DOS. Figure 2 shows this quantity for $U=2.0775 \mathrm{eV}$, just below $U_{c}$. The region $U<U_{c}$ is the band insulating regime, its DOS being similar to the noninteracting $(U=0)$ one, i.e., a branch cut surrounded by two singularities and cut by a gap at the middle. Figure 3 , on the

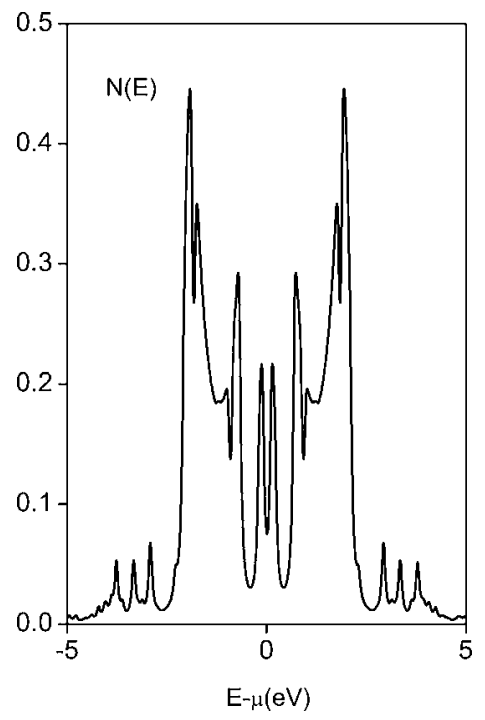

FIG. 3. The same as Fig. 2 but for $U=2.0780$, just above $U_{c}$.

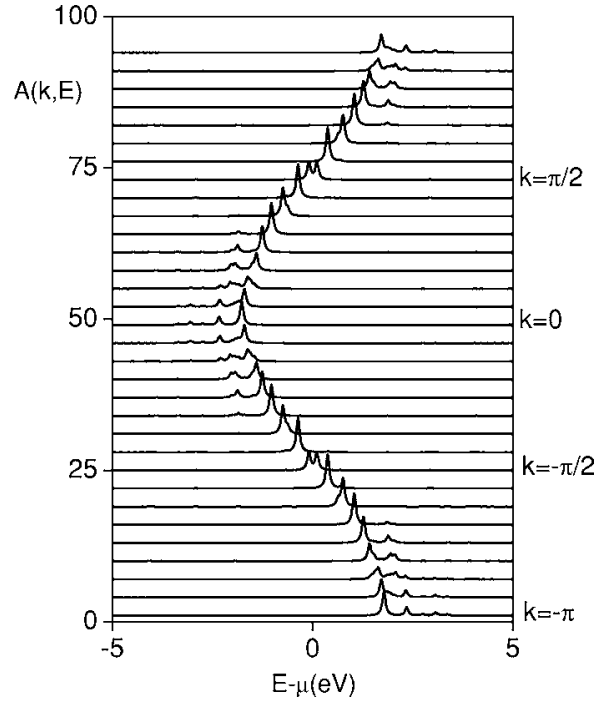

FIG. 4. Spectral-weight function $A(k, E)$ for the same situation of Fig. 2. An offset has been given to the plots for different $k$ 's along the large Brillouin zone $(-\pi \leqslant k<\pi)$ in order to avoid superposition. The figures along the left vertical axis count the number of $k$ 's starting from $-\pi$. On the right vertical axis, some special $k$ 's are indicated

other hand, displays the DOS for $U=2.0780 \mathrm{eV}$, just above $U_{c}$, in correspondence with the third panel (from top to bottom) of Fig. 1. The two outer peaks in this latter figure mark the edges of the gap between the Hubbard-like bands which are now considerably narrower than in the first regime. The two inner peaks stand isolated near the chemical potential, resembling "impuritylike," nondispersive peaks inside the gap. All through the region $U>U_{c}$, the DOS is quite different from the noninteracting one. Notice the change in scale from Fig. 2 to Fig. 3. A huge transfer of weight has taken place from the low-energy part of the continuum to the highenergy peaks surrounding the branch cuts as well as to the four peaks inside the gap. Hence other $k \neq k_{F}$ play an important role once again.

To see the changes occurring at $k \neq \pm \pi / 2$, Figs. 4 and 5 display $A(k, E)$ along the large Brillouin zone (BZ) $-\pi \leqslant k$ $<\pi$ (in the extended zone scheme). The portion $|k|>\pi / 2$ can be mapped, if one wishes, into the small BZ. An offset has been provided to the plots for different $k$ 's in order to avoid superposition. The figures along the left vertical axis number the wave vectors starting from $k=-\pi$, only a selected set of them (32) having been drawn for clarity. Figure 4 should be compared with the DOS of Fig. 2 and shows a cosinelike band cut by a gap at the Fermi points (cf. the second panel in Fig. 1). Two shadow bands covering only part of the BZ are clearly visible around $k=0$ (occupied) and $k=\pi$ (empty). They give rise to the side peaks outside the main body of the corresponding DOS. Figure 5 should now be confronted with the DOS of Fig. 3. The cosinelike band is now cut by a wider gap delimited by the outer peaks at $k_{F}$ $= \pm \pi / 2$ (cf. Fig. 1, third panel). The inner peaks give rise to weakly dispersive, flat bands covering only a small portion of the $\mathrm{BZ}$ around $k_{F}$. The shadow bands around $k=0$ and $\pi$ have now almost disappeared along with the side peaks, rather weak, in the DOS. 


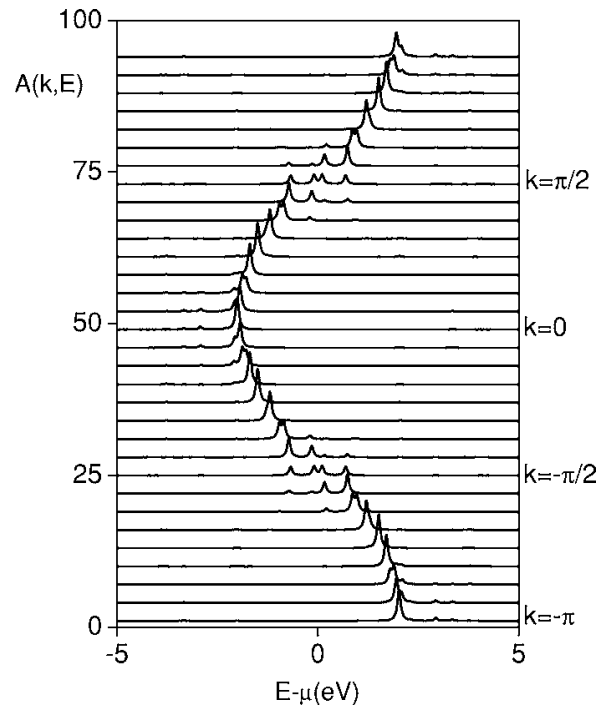

FIG. 5. Same as Fig. 4, but for $U=2.0780$, just above $U_{c}$.

\section{Discussion}

Outside the gap region, the band structures in Figs. 4 and 5 can be better understood by considering the IHM as a two-component system made up of an electron-doped (the odd sites) and a hole-doped (the even sites) system, both slightly away from half filling. Then the band structure around $k=0$ is very reminiscent of the hole-doped Hubbard model (a spinon and a holon band). Likewise, the band structure around $k=\pi$ resembles that of the electron-doped Hubbard model, with the same interpretation. In the gap region, on the other hand, the physics is essentially controlled by the separated atoms limit, as explained at the end of Sec. III A. Since, however, a range of $k$ 's around $k_{F}$ also contributes to the DOS inside the gap, the relative strength of the inner and outer peaks in Fig. 5 need not be that derived from $k_{F}$ alone.

The transfer of spectral weight at $U_{c}$ is in fact the only signature of a quantum phase transition detected by the single-particle GF. All the $E$-integrated quantities like the ground-state energy, the ionicity, the double occupancy, and the bond charge are continuous functions of $U$, even at $U_{c}$, and thus do not provide any hint of a quantum phase transition. This is, for instance, the case of the bond charge $n_{i j}$ which tells us nothing about a spontaneous dimerization. It is continuous across any single site, e.g., $n_{23}=n_{21}$ and not $n_{23}$ $\neq n_{21}$. To settle this issue one must turn to bond-bond correlations, which have been extensively studied recently by several authors. ${ }^{4-10}$ These correlations tell us that our second phase is indeed spontaneously dimerized and therefore the detected transition may correspond to the critical point of the well-known two-point scenario. The second critical point, associated with the spin gap, cannot be detected by the SWF. We wish to stress here that our results are not in contradiction with those obtained by these authors regarding the nature of quantum phase transitions in the IHM. Simply, quantities like excitation gaps, spin gaps, bond-bond correlations, etc. are not directly accessible to the single-particle GF. Our results rather add information about a quantum phase transition associated with the single-particle gap minimum.

\section{COMPARISON WITH Q1D MATERIALS}

We want to demonstrate now that both regimes are found in ARPES of Q1D materials in their insulating phase. These materials have been the object of intense experimental and theoretical activity over the last 20 years. They show highly anisotropic properties, with a privileged direction of enhanced charge transport. Their interest from the theoretical standpoint lies in the hope that they can be good candidates for the physical realization of non-Fermi-liquid behavior. This interest, in low-D systems in general, has expanded very rapidly in recent years partly due to the technological development of low-D artificial structures and nanoscale materials.

Above their Peierls temperature (or when doped away from half filling), these Q1D systems are conductors and display Luttinger liquid behavior, ${ }^{15}$ i.e., the absence of quasiparticle excitations in the Fermi liquid sense (a quasiparticle peak at the Fermi level) and the excitation, instead, of decoupled collective modes of charge (holons) and spin (spinons) character, a phenomenon usually known as chargespin separation. The absence of a Fermi edge has indeed been found in ARPES of the inorganic compounds $\left(\mathrm{TaSe}_{4}\right)_{2} I$ (Refs. 16 and 17) and $\mathrm{K}_{0.3} \mathrm{MoO}_{3},{ }^{18}$ as well as the organic conductor TTF-TCNQ (tetrathiafulvalene-tetracyanoquinodimethane). ${ }^{19,20}$ For a good review, see Ref. 21. Clear experimental signatures of spin-charge separation are, however, very scarce in Q1D conductors, with the notable exception of TTF-TCNQ reported very recently. ${ }^{20}$ All these compounds in their metallic state can be modeled by the low-energy physics of the doped 1D single-band Hubbard Hamiltonian. ${ }^{20}$ Alternatively, they have been analyzed on the basis of the Luttinger model or the Luther-Emery model (when a spin gap is expected). Some puzzles still remain unsolved. ${ }^{21}$

Below their Peierls temperature, these Q1D compounds, as well as many others like halogen-bridged transition-metal chains, conjugated polymers, and organic charge-transfer salts, are usually insulating, having nonmetallic ground states with or without charge-density waves (CDWs). Thus $\left(\mathrm{TaSe}_{4}\right)_{2} \mathrm{I}$ and the blue bronze $\mathrm{K}_{0.3} \mathrm{MoO}_{3}$, for instance, are CDW insulators, ${ }^{21}$ while $\mathrm{SrCuO}_{2}$ and $\mathrm{Sr}_{2} \mathrm{CuO}_{3}$ are chargetransfer insulators. ${ }^{22}$ Signatures of spin-charge separation have also been found in ARPES of $\mathrm{SrCuO}_{2},{ }^{23}$ and in the dielectric response of $\mathrm{Sr}_{2} \mathrm{CuO}_{3}{ }^{24}$

Since most of these materials have inequivalent sites (cations and anions), the 1D IHM offers a better starting point for modeling them than the simplest, one-band Hubbard model. Both metallic and insulating phases can be accommodated by varying the electron filling. Instead of embarking on a detailed comparison of ARPES and $A(k, E)$ for different Q1D materials, let us take, just for illustrative purposes, $\left(\mathrm{TaSe}_{4}\right)_{2} I$ and $\mathrm{K}_{0.3} \mathrm{MoO}_{3}$. Since they have occupied bands of width roughly $1 \mathrm{eV}$, we have accordingly taken $t=-0.5 \mathrm{eV}$ and $\Delta=1 \mathrm{eV}$. The critical $U$ separating both regimes is now $U_{c}=2 \mathrm{eV}$. Figure 6 shows $A(k, E)$ between $k=0$ and $\pi / 2$ for $U=0.5 \mathrm{eV}<U_{c}$, a case of the first regime, with a single occupied peak at $k=\pi / 2$. Notice that $U$ has been fitted to give the correct gap at $k=\pi / 2$. This one-band structure resembles 


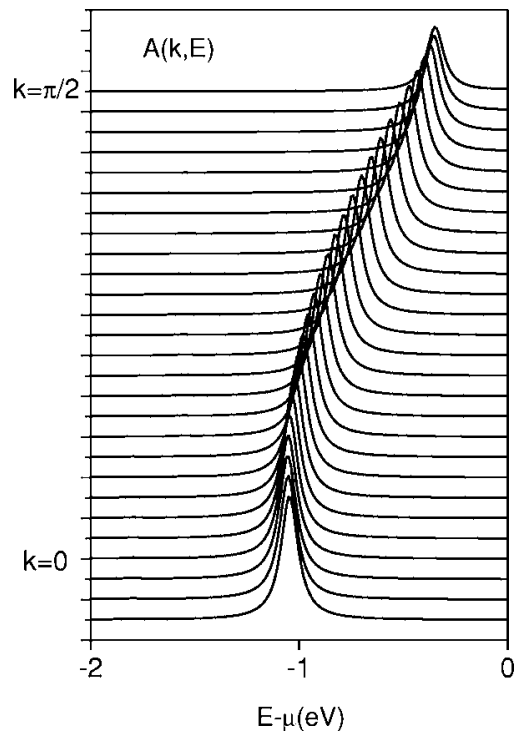

FIG. 6. Spectral-weight function for (in eV) $t=-0.5, \Delta=1$, and $U=0.5$.

that of $\left(\mathrm{TaSe}_{4}\right)_{2} I$ in the direction parallel to the chain [compare with Fig. 2(a) of Ref. 17). Likewise, Fig. 7 shows the same information as Fig. 6, but for $U=2.1 \mathrm{eV}>U_{c}$. We now see a two-band structure in the neighborhood of $k=\pi / 2$. This resembles the band structure of $\mathrm{K}_{0.3} \mathrm{MoO}_{3}$ (compare with Fig. 15 of Ref. 18). The flat band is clearly visible at the top right of Fig. 7. However, notice the presence of a strong (holon) shadow band in this figure $\left(U>U_{c}\right)$ instead of in Fig. $6\left(U<U_{c}\right)$, as one would expect from our discussion in Sec. III C. A rationale for this behavior is that the $U=0.5 \mathrm{eV}$ used

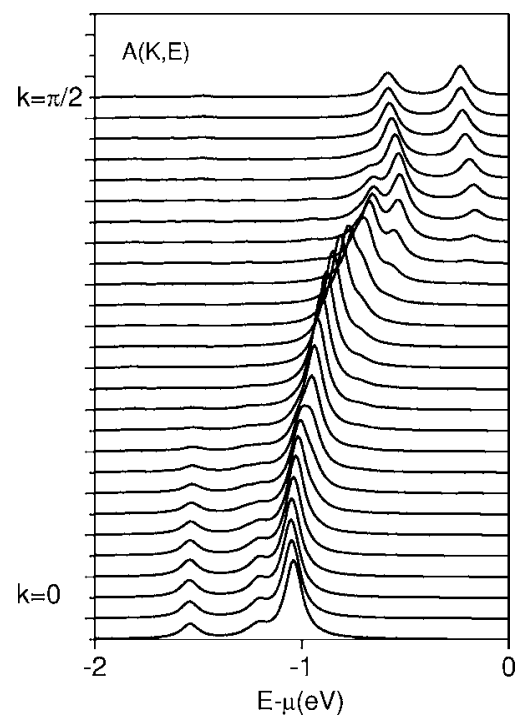

FIG. 7. The same as Fig. 6, but for $U=2.1 \mathrm{eV}$. in Fig. 6 is small and very far from $U_{c}$, whereas the $U$ of Fig. 7 is $2.1 \mathrm{eV}$, just above $U_{c}$, and yields slightly doped sublattices away from half filling. Holon bands tend to be stronger near (but not at) half filling.

\section{CONCLUDING REMARKS}

Using the cluster perturbation theory approach of Senechal et al., ${ }^{11,13}$ we have calculated the single-particle spectral-weight function $A(k, E)$ of the ionic 1D Hubbard model at half filling. A quantum phase transition is found at a critical value of $U, U_{c}(t, \Delta)$, which depends on both the hopping amplitude $t$ and the on-site energy difference $\Delta$ between even and odd sites. It is signaled by a large transfer of spectral weight at $k_{F}$ from the inner to the (weak) outer peaks leading to a clear four-peak structure for $U>U_{c}$. As one moves away from $k_{F}$, the inner peaks are found to delimit a gap which decreases from its initial value $\Delta$ (at $U=0)$ down to a small, but nonvanishing, value at $U_{c}$, and then increases again for $U>U_{c}$. In this region, the inner peaks turn into flat, almost nondispersive bands which cover only a small portion of the BZ and push further apart the wider bands of the band-insulating regime. This is accomplished by a huge transfer of weight from the low-energy portion of the continuum into the gap region (the inner peaks and the band edges). This is quite visible by comparing the DOS of Figs. 2 and 3. The new situation can alternatively be described in terms of a wider gap between the Hubbard-like bands plus two impuritylike peaks inside the gap. This is somewhat reminiscent of the periodic Anderson model. As $U$ increases further, the flat bands approach the Hubbard bands, finally merging into them. Asymptotically, a Mott-Hubbard situation is approached in a continuous way, without any signature of abrupt change of $A(k, E)$ in this region of large $U$.

The GF does not give any hint of the nature of the second, strong-coupling phase, since all the $E$-integrated quantities are continuous. For instance, the band charge is quite symmetric around any site, i.e., $n_{23}=n_{21}$, thus giving no information about a spontaneously dimerized phase. This information comes from bond-bond correlations which have been extensively studied in the past. The single-particle GFs only tell us that there is a quantum phase transition when the simple-particle charge gap has a minimum. However, since bond-bond correlations do not vanish for $U>U_{c}$, we can say that the strong-coupling phase is spontaneously dimerized. The transition detected by the GF, therefore, must correspond to the first critical point in the two-critical-point scenario. The second critical point, associated with the spin gap, cannot be detected by the GF. Let us finally end by saying that different Q1D materials in their insulating phase have band structures which can be classified as lying in either the first or the second regime.

\section{ACKNOWLEDGMENT}

We acknowledge the financial support of the Spanish DGICYT through Project No. BFM 2002-01594. 
*Electronic address: refolio@imaff.cfmac.csic.es

${ }^{1}$ N. Nagaosa and J. Takimoto, J. Phys. Soc. Jpn. 55, 2735 (1986).

${ }^{2}$ T. Egami, S. Ishihara, and M. Tachiki, Science 261, 1307 (1993).

${ }^{3}$ S. Ishihara, T. Egami, and M. Tachiki, Phys. Rev. B 49, 8944 (1994).

${ }^{4}$ R. Resta and S. Sorella, Phys. Rev. Lett. 74, 4738 (1995).

${ }^{5}$ T. Wilkens and R. Martin, Phys. Rev. B 63, 235108 (2001).

${ }^{6}$ A. P. Kampf, M. Sekania, G. I. Japaridze, and Ph. Brune, J. Phys.: Condens. Matter 15, 5895 (2003).

${ }^{7}$ M. Fabrizio, A. O. Gogolin, and A. A. Nersesyan, Phys. Rev. Lett. 83, 2014 (1999).

${ }^{8}$ Y. Takada and M. Kido, J. Phys. Soc. Jpn. 70, 21 (2001).

${ }^{9}$ Y. Z. Zhang, C. Q. Wu, and H. Q. Lin, Phys. Rev. B 67, 205109 (2003).

${ }^{10}$ S. R. Manmana, V. Meden, R. M. Noack, and K. Schonhammer, Phys. Rev. B 70, 155115 (2004).

${ }^{11}$ D. Senechal, D. Perez, and M. Pioro-Ladriere, Phys. Rev. Lett. 84, 522 (2000).

${ }^{12}$ E. Dagotto, Rev. Mod. Phys. 66, 763 (1994).

${ }^{13}$ D. Senechal, D. Perez, and D. Plouffe, Phys. Rev. B 66, 075129 (2002).

${ }^{14}$ M. Potthoff, M. Aichhorn, and C. Dahnken, Phys. Rev. Lett. 91, 206402 (2003).

${ }^{15}$ F. D. M. Haldane, J. Phys. C 14, 2585 (1981).
${ }^{16}$ Y. Hwu, P. Almras, M. Marsi, H. Berger, F. Levy, M. Grioni, D. Malterre, and G. Margaritondo, Phys. Rev. B 46, R13624 (1992).

${ }^{17}$ A. Terrasi, M. Marsi, H. Berger, G. Margaritondo, R. J. Kelley, and M. Onellion, Phys. Rev. B 52, 5592 (1995).

${ }^{18}$ G. H. Gweon, J. D. Denlinger, J. W. Allen, R. Claesson, C. G. Olson, H. Hochet, J. Marcus, C. Schlenker, and L. F. Schneemeyer, J. Electron Spectrosc. Relat. Phenom. 117-118, 481 (2001).

${ }^{19}$ F. Zwick, D. Jerome, G. Margaritondo, M. Onellion, J. Voit, and M. Grioni, Phys. Rev. Lett. 81, 2974 (1998).

${ }^{20}$ M. Sing, U. Schwingenschlogl, R. Claesson, P. Blaha, J. M. P. Carmelo, L. M. Martelo, P. D. Sacramento, M. Dressel, and C. S. Jacobsen, Phys. Rev. B 68, 125111 (2003).

${ }^{21}$ M. Grioni and J. Voit, in Electron Spectroscopies Applied to LowDimensional Materials, edited by H. Starnberg and H. Hughes (Kluwer, Dordrecht, 2000), Vol. 1.

${ }^{22}$ K. Penc and W. Stephan, Phys. Rev. B 62, 12707 (2000).

${ }^{23}$ C. Kim, A. Y. Matsuura, Z-X. Shen, N. Motoyama, H. Eisaki, S. Uchida, T. Tohyama, and S. Maekawa, Phys. Rev. Lett. 77, 4054 (1996).

${ }^{24}$ R. Neudert, M. Knupfer, M. S. Golden, J. Fink, W. Stephan, K. Penc, N. Motoyama, H. Eisaki, and S. Uchida, Phys. Rev. Lett. 81, 657 (1998). 